\title{
On an elementary proof of Euler's product expansion for the sine
}

\author{
Anonymous
}

April 27, 2019

\begin{abstract}
Using, elementary trigonometric identities, we give a proof of the Euler's product expansion for the sine.
\end{abstract}

Euler's infinite product for the sine is, for $x \in \mathbb{R}$, the identity

$$
\frac{\sin x}{x}=\prod_{j=1}^{\infty}\left(1-\frac{x^{2}}{j^{2} \pi^{2}}\right) \text {. }
$$

In the monthly article [1], the author gives an elementary proof of the Euler's product expansion for the sine using a trigonometric identity and Tannery's theorem for infinite products. However, the author also makes use of the Wallis's product formula. Our proof here makes use of trigonometric identities without the usage of Wallis's product formula, which is often, obtained from the Euler's product expansion for the sine.

We begin with the following identity.

Theorem 1. For all complex numbers $z$ and all integers $n$, we have

$$
\sin n z=2^{n-1} \sin z \sin \left(z+\frac{\pi}{n}\right) \sin \left(z+\frac{2 \pi}{n}\right) \cdots \sin \left(z+\frac{(n-1) \pi}{n}\right) .
$$

Proof. We can write

$$
\begin{array}{r}
\sin n z=\frac{e^{n i z}-e^{-n i z}}{2 i}=\frac{e^{-n i z}\left(e^{2 n i z}-1\right)}{2 i} \\
=\frac{e^{-n i z}\left(e^{2 i z}-1\right)\left(e^{2 i z}-e^{\frac{-2 i \pi}{n}}\right) \cdots\left(e^{2 i z}-e^{\frac{-2(n-1) i \pi}{n}}\right)}{2 i} \\
=k \cdot \sin z \sin \left(z+\frac{\pi}{n}\right) \sin \left(z+\frac{2 \pi}{n}\right) \cdots \sin \left(z+\frac{(n-1) \pi}{n}\right)
\end{array}
$$

where

$$
k=(2 i)^{n-1} e^{\frac{-i \pi}{n}(1+2 \cdots+(n-1))}=(2 i)^{n-1} e^{\frac{-i \pi(n-1)}{2}}=2^{n-1} .
$$

Corollary 1. For all integers $n$, we have

$$
\sin \frac{\pi}{n} \sin \frac{2 \pi}{n} \cdots \sin \frac{(n-1) \pi}{n}=\frac{n}{2^{n-1}} .
$$

Proof. To obtain the result we divide both sides (1) by $\sin z$ and let $z \rightarrow 0$.

Now, letting $n=2 m+1$ in (1), we get

$$
\begin{array}{r}
\sin n z=2^{n-1} \prod_{j=0}^{2 m} \sin \left(z+\frac{j \pi}{n}\right)=2^{n-1}(-1)^{m} \prod_{j=0}^{m} \sin \left(z+\frac{j \pi}{n}\right) \prod_{j=m+1}^{2 m} \sin \left(z+\frac{j \pi}{n}-\pi\right) \\
=2^{n-1}(-1)^{m} \prod_{j=-m}^{m} \sin \left(z+\frac{j \pi}{n}\right) \\
=(-1)^{m} 2^{n-1} \sin z \prod_{j=1}^{m} \sin \left(z+\frac{j \pi}{n}\right) \sin \left(z-\frac{j \pi}{n}\right) \\
=(-1)^{m} 2^{n-1} \sin z \prod_{j=1}^{m}\left(\sin ^{2} z-\sin ^{2} \frac{j \pi}{n}\right)
\end{array}
$$


Now, from equation (2) we can conclude $\prod_{j=1}^{m} \sin ^{2} \frac{j \pi}{n}=\frac{n}{2^{n-1}}$. Using this and replacing $z$ by $z / n$, we obtain

$$
\sin z=n \sin \frac{z}{n} \prod_{j=1}^{m}\left(1-\frac{\sin ^{2} \frac{z}{n}}{\sin ^{2} \frac{j \pi}{n}}\right) .
$$

Next, we state and use the Tannery's theorem for infinite products

Theorem 2 (Tannery's theorem for infinite products, [2]). For all natural numbers $n \in \mathbb{N}$, let $\prod_{j=1}^{\infty}\left(1+a_{j}(n)\right)$ be a convergent infinte product. If for all $j$, $\lim _{n \rightarrow \infty} a_{j}(n)=a_{j}$ and $\left|a_{j}(n)\right| \leq M_{j}$ for all $n$, where the series $\sum M_{j}$ converges, then

$$
\lim _{n \rightarrow \infty} \prod_{j=1}^{\infty}\left(1+a_{j}(n)\right)=\prod_{j=1}^{\infty}\left(1+a_{j}\right)
$$

Now, let

$$
a_{j}(n)=\frac{-\sin ^{2} \frac{z}{n}}{\sin ^{2} \frac{j \pi}{n}}
$$

Now, since, for $0 \leq x \leq \pi / 2$,

$$
\frac{\sin x}{x} \geq 1-\frac{x^{2}}{6} \geq \frac{1}{2}
$$

we have, for $1 \leq j \leq n / 2$,

$$
n \sin \frac{j \pi}{n} \geq \frac{j \pi}{2}
$$

that is,

$$
\frac{1}{n^{2} \sin ^{2} \frac{j \pi}{n}} \leq \frac{4}{j^{2} \pi^{2}}
$$

But, since,

$$
\sin ^{2} \frac{z}{n} \leq \frac{z^{2}}{n^{2}} \leq \frac{l^{2}}{n^{2}}
$$

where $l$ be the constant $>z$ for given real $z$. Thus,

$$
\left|a_{j}(n)\right|=\frac{\sin ^{2} \frac{z}{n}}{\sin ^{2} \frac{j \pi}{n}} \leq \frac{4 l^{2}}{j^{2} \pi^{2}} .
$$

Now, using the Tannery's theorem for infinite products, we can write

$$
\sin z=z \lim _{n \rightarrow \infty} \prod_{j=1}^{\infty}\left(1-\frac{\sin ^{2} \frac{z}{n}}{\sin ^{2} \frac{j \pi}{n}}\right)=z \prod_{j=1}^{\infty} \lim _{n \rightarrow \infty}\left(1-\frac{\sin ^{2} \frac{z}{n}}{\sin ^{2} \frac{j \pi}{n}}\right)=z \prod_{j=1}^{\infty}\left(1-\frac{z^{2}}{j^{2} \pi^{2}}\right) .
$$

REFERENCES

[1] Óscar Ciaurri (2015) Euler's Product Expansion for the Sine: An Elementary Proof, The American Mathematical Monthly, 122:7, 693-695, DOI: 10.4169/amer.math.monthly.122.7.693

[2] Paul Loya, Real Analysis 1 notes, Page 322,

Link: http://people.math.binghamton.edu/dikran/478/Ch6.pdf. 